Relations industrielles

Industrial Relations

\title{
Labor Relations in the Netherlands, par John P. Windmuller, Cornell University Press, Ithaca, New York, 1969, 469 pages.
}

Jean Sexton

Volume 24, numéro 2, 1969

URI : https://id.erudit.org/iderudit/028037ar

DOI : https://doi.org/10.7202/028037ar

Aller au sommaire du numéro

Éditeur(s)

Département des relations industrielles de l'Université Laval

ISSN

0034-379X (imprimé)

1703-8138 (numérique)

Découvrir la revue

Citer ce compte rendu

Sexton, J. (1969). Compte rendu de [Labor Relations in the Netherlands, par John P. Windmuller, Cornell University Press, Ithaca, New York, 1969, 469 pages.] Relations industrielles / Industrial Relations, 24(2), 456-456.

https://doi.org/10.7202/028037ar

Tous droits réservés @ C Département des relations industrielles de l'Université Laval, 1969
Ce document est protégé par la loi sur le droit d'auteur. L’utilisation des services d'Érudit (y compris la reproduction) est assujettie à sa politique d'utilisation que vous pouvez consulter en ligne.

https://apropos.erudit.org/fr/usagers/politique-dutilisation/ 
Labor Relations in the Netherlands, par John P. Windmuller, Cornell University Press, Ithaca, New York, 1969, 469 pages.

On a très peu étudié le système hollandais de relations industrielles excepté, peut-être, pour ses politiques de salaires d'après-guerre qu'on ne peut comprendre complètement sans connaître le contexte historique et institutionnel de ce pays. Le but de ce livre est de combler cette lacune en présentant le système de relations industrielles hollandais tant sous son aspect historique qu'institutionnel.

Pour ceux qui ne connaîtraient pas l'histoire générale et le régime politique propres à la Hollande, l'auteur a cru bon donner quelques références utiles en guise de préface.

Partiellement basé sur le modèle de Dunlop, le professeur Windmuller aborde des sujets tels l'histoire du mouvement syndical, la structure et les activités des syndicats d'aujourd'hui, la structure et le rôle des associations d'employeurs et le rôle des comités paritaires consultatifs dans l'établissement des politiques tant au plan national qu'au niveau de l'industrie et de l'entreprise. Une partie importante de cet ouvrage est consacrée aux origines, au fonctionnement et aux réalisations de la négociation collective centralisée en insistant plus particulièrement sur les politiques de salaires d'après-guerre. L'auteur a cru bon ajouter aux textes un bon nombre de tableaux statistiques illustrant les résultats de ce système de relations industrielles. En plus, le professeur Windmuller compare sous plusieurs aspects le système hollandais de relations industrielles avec les expériences de quelques autres pays.

Labor Relations in the Netherlands est une contribution valable à l'étude des systèmes comparés de relations industrielles.
The Traditional Trade of Asia, by G.G.F. Simkin, London, Oxford University Press, 1968, 417 pages.

A proprement parler, c'est une histoire des civilisations d'Asie que nous présente le professeur Simkin, une histoire quil approche par l'étude historique du commerce et de ses développements en Asie. En effet, le commerce, l'échange a mis en contact pendant plusieurs centaines d'années les différentes entités nationales asiatiques, non seulement entre elles, mais aussi avec l'Occident. A l'occasion de l'échange, non seulement les particularités économiques des peuples sont mises en présence, mais aussi leur culture, leurs organisations politiques, leurs manières de vivre et de travailler. Songeons aux magnifiques exemples qui nous sont donnés par l'auteur à l'occasion de la pénétration des Grecs ou des Européens dans les marchés d'Orient.

Bien qu'il soit économiste, M. Simkin en vint donc à utiliser de multiples approches pour traiter ce sujet; il doit se faire tantôt politicologue et sociologue, tantôt historien et géographe pour mener à bien son étude. Même s'il se défend bien d'avoir rien apporté de neuf dans ces domaines, accordons lui un grand respect pour le travail de collection des informations et de leur présentation. Accordons lui aussi la reconnaissance d'un effort analytique extrêmement: poussé afin de tirer de l'histoire commerciale de l'Asie des grandes tangentes économiques, politiquement sociologiques qui déterminent encore le développement de l'Asie.

Enfin, signalons que tous auront intérêt à consulter à maintes occasions un ouvrage de ce genre, soit pour y trouver des faits historiques, des données objectives, des analyses socio-politiques, soit pour y trouver l'exemple d'une analyse économique voulue dépendante des autres sciences de l'homme et menée avec un souci propre aux grands chercheurs. 